\title{
Volume holographic interconnections with maximal capacity and minimal cross talk
}

\author{
Hyuk Lee \\ Polytechnic University, Department of Electrical Engineering, Brooklyn, New York 11201 \\ Xiang-guang Gu and Demetri Psaltis \\ California Institute of Technology, Department of Electrical Engineering, Pasadena, California 9125
}

(Received 1 July 1988; accepted for publication 2 November 1988)

\begin{abstract}
Optical interconnections utihizing volume holography are described. Intrinsic cross-talk effects that limit the number of independent interconnections are identified and analyzed by applying coupled-wave analysis. Sampling grids for removing the first-order cross tak are presented, resulting in a system limited by second-and third-order cross talk only.
\end{abstract}

\section{INTRODUCTBON}

Optical interconnecting elements that exploit free-propagating light waves can potentially act as a powerful alternative to electrical wiring because free-propagating photons lack the interactive nature of electrons. ' Optical interconnections can be particularly useful for the optical implementation of neutral computers ${ }^{2}$ in which each processing element is interconnected to many others (typically several thousand). As an example, a network that is capable of processing images may consist of several million processing elements (or "neurons") and therefore there is a very large number of interconnections to be specified in such a system. If the system that is utilized to simulate all these connections is planar (e.g., electronic or an optical system that utiizes a planar medium to specify the connectivity partern), then the area of the device grows in proportion to the total number of connections. As an example, let us assume that the area required to record the strength of each interconnection is 10 $\mu \mathrm{m}^{2}$, then the total area required to simulate a network that is comprised of $10^{9}$ connections is $10 \times 10 \mathrm{~cm}^{2}$. This makes the fabrication of such a device very dificult and, in the case of the optical implementation, the size of the optical system becomes exceedingly large. To overcome this shortcoming, we have previously proposed ${ }^{3}$ a holographic optical interconnection method for utilizing a three-dimensional storage medium which provides a much higher storage density. In this paper, we derive the interconnection pattern having minimum cross talk and the signal-to-noise ratio for this interconnecting configuration.

\section{HOLOGRAPHIC INTERCONNECTIONS}

To identify the fundamental cross-talk effects that limit the available number of independent interconnections, a global volume holographic interconnection between $N$ input and $N$ output pixels is considered. The arrangement we will be using is shown in Fig. 1. The input and output pixels are arranged in planes. A lens collimates light from each input point and therefore the light incident on the crystal in Fig. 1 due to a single point at the input is a plane wave whose propagation direction is determined by the position of the pixel. Similarly, an output lens focuses each difracted plane wave to a pixel on the output plane. The interconnection between each pair of input-output points is performed by a separate grating, with the strength of each grating determining the weight of the connection. Each grating can be recorded with a separate exposure which would require a total of $N^{2}$ exposures. We can reduce the number of required exposures by forming $N$ multiple holographic exposures ${ }^{4}$ as follows. One input point is turned on during each exposure and the desired connectivity pattern between the selected input point and all the output points is recorded at the training plane (see Fig. 1). An exposure of the interference pattern between the two waves is recorded and the process is repeated for each of the $N$ input points. If we neglect diffaction effects at the crystal boundaries, then the interconnection pattern consists of perfect sinusoidal gratings, which include: (1) $N(N-1) / 2$ gratings that are recorded by the interference between pixels that are simultaneously on at the training plane during the recording; and (2) $N^{2}$ gratings connecting input and output pixels. For convenience, the former set of $N(N-1) / 2$ gratings are referred to as intralayer gratings and the latter set of $N^{2}$ gratings are described as interlayer gratings.

An independent interconnection is defined in such a way that the intensity $I_{p}$ of the diffracted light wave at the output pixel $p$ is given by

$$
I_{p}=\sum_{i=1}^{N} \eta_{p i} I_{i}
$$

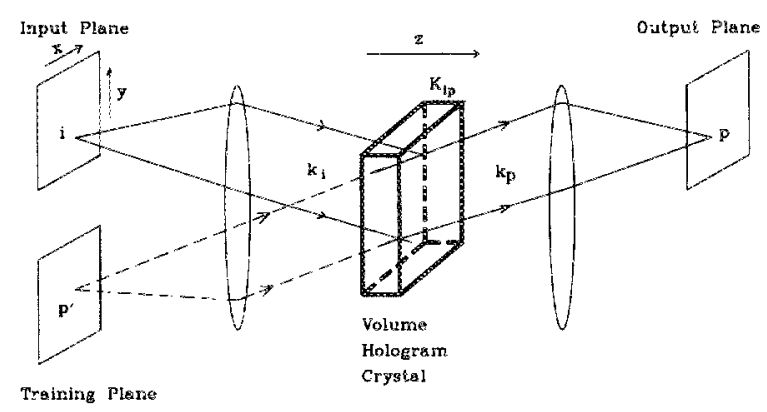

FIG. 1. Illustration of the proposed interconnection scheme between an input point $i$ and an output point $p$. One grating is stored by interfering the two beams coming from point sources $i$ and $p^{\prime}$ after passing through a Fourier transforming lens. Point $p^{\prime}$ is the inverted image of point $p$. After storing the grating, light coming from point $i$ is diffracted by the grating and focused on point $p$. Therefore, the stored grating interconnects points $i$ and $p$. 
where $i$ is the index that represents input pixels, $I_{i}$ is the intensity at the input pixel $i$, and $\eta_{p i}$ is the diffraction effciency of the grating generated by the interference between the input pixel $i$ and the pixel $p^{\prime}$ at the training plane that corresponds to the output pixel $p$. We have assumed in Eq. (1) that the read-out light is spatially incoherent. This means that the light intensity reaching each output point is a Iinear combination of the light intensities of the input pixels and therefore we have used light intensity as the variable that describes the system. If the hologram is read-out with spatially coherent light, then the field is the appropriate variable to use. The field is a complex quantity (has both amplitude and phase) and therefore the colserent case is generally more difficult to analyze and also implement.

The cross-talk effect in volume holographic interconnections is defined as the difference between the actual light intensity $\widetilde{I}_{p}$ obtained at the pixel $p$ and the desirable intensity of Eq. (1). If we consider only first-order cross talk (i.e., neglecting the contribution of multiple diffraction), we can write $\widetilde{I}_{p}$ as follows:

$$
\tilde{I}_{p}=\sum_{i} \eta_{p i} I_{i}+\sum_{j \neq i} \sum_{i \neq p} \sum_{i} \eta_{i p j l} I_{i}
$$

where $\eta_{i p j !}$ is the diffraction efficiency with which light is diffracted from pixel $i$ to pixel $p$ due to a grating that was recorded by pixel $j$ at the input and $l$ at the output. Coupledwave analysis ${ }^{5}$ is utilized below to evaluate those cross-talk effects. For this purpose small difiraction eficiency for an individual grating is assumed. Due to the assumption of small diffraction efficiency, the primary cross-talk effect can be evaluated by means of a first-order coupled-wave analysis. In such an analysis, an input light wave at pixel $i$ interacts with every grating in the volume hologram independently and without an intermediate rediffraction. Diffracted light waves from the intralayer gratings do not contribute to cross-talk effects because of large phase mismatch, and therefore we only need to consider the first-order cross talk that results from the interlayer gratings.

Let us consider an output pixel $p$. The light intensity received at $p$ including the first-order cross-talk effect is given by Eq. (2). The cross-talk diffraction efficiency $\eta_{i p j !}$ calculated from coupled mode analysis is approximately ${ }^{5}$

$$
\eta_{i p j l}= \begin{cases}\eta_{j l} \sin c^{2}\left(\Delta k_{i p j l} L / 2 \pi\right) & \text { if }(2 \pi / \lambda) \mathbf{n}_{i}+\mathbf{K}_{j l}=\mathbf{n}_{p}\left\|(2 \pi / \lambda) \mathbf{n}_{i}+\mathbf{K}_{j l}\right\| \\ 0 & \text { otherwise, }\end{cases}
$$

where $n_{i}$ and $n_{p}$ denote unit vectors in the direction of propagation from the input pixel $i$ and towards the output pixel $p$, respectively. $L$ is the thickness of the crystal and $\Delta k_{i p i j}$ denotes the phase mismatch for the interaction between the grating $\mathrm{K}_{j l}$ that has been recorded for interconnecting point $j$ and to point $l$ and the optical wave emanating from the input pixel $i$ and it is given by

$$
\Delta k_{i p j l}=\|(2 \pi / \lambda)\left(\mathbf{n}_{i}-\mathbf{n}_{p}\right)+\mathbf{K}_{j l} \mid \cdot
$$

$\lambda$ is the optical wavelength in the crystal.

\section{FIRST-ORDER CROSS TALK}

First-order cross talk can be eliminated if one can arrange input and output pixels so that all the $N^{2}$ gratings in Eq. (2), except for the grating $\mathbf{K}_{\text {ip }}$ yielding the signal, belong to one of the two types of gratings defined below. The first type is characterized by the condition that the phase mismatch given by Eq. (4) is larger than $2 \pi / L$, in which case the diffracted light intensity is very small; for these gratings, the first-order cross-talk effect can be neglected. The second type consists of gratings for which the diffracted light waves do not propagate to any one of the output pixels used for the interconnection, in which case from Eq. (3) we have the $\eta_{i p j l}=0$ and hence such gratings do not contribute any light intensity at the pixel $p$ through first-order cross talk. To derive an arrangement that will ensure that all the recorded gratings satisfy one of the two conditions stated above, we note that the phase mismatch described by Eq. (4) is determined by the geometry of the input and output pixels. The wave vector diagram is drawn in Fig. 2 , where $\mathrm{k}_{i}$ and $\mathrm{k}_{j}$ refer to the input wave vectors and $k_{p}$ and $k_{l}$ are the output wave vectors. The condition

$$
\mathbf{n}_{p}=\left(\mathbf{k}_{i}+\mathbf{K}_{j l}\right) /\left(\left\|\mathbf{k}_{i}+\mathbf{K}_{j l}\right\|\right)
$$

states that the unit vector $\mathrm{n}_{p}$ is the direction of the vectorial sum of the input vector $k_{i}$ and the grating vector. This indicates that the grating $\mathbf{K}_{j l}$ is a grating of the first type, being capable of diffracting light from $i$ to $p$ unless it is phase mismatched. Therefore, once a pair $(i, p)$ is selected it is imperative that all the remaining points $(j, l)$ are selected such that if Eq. (5) is satisfied then $\Delta k_{i p j l}$ is bigger than $2 \pi / L$. The degeneracy condition that must be avoided is

$$
\Delta k_{i p j l}=\left\|\mathbf{k}_{i}+\mathbf{K}_{j l}-\mathbf{k}_{p}\right\|<2 \pi / L .
$$

This condition specifies two strips on the k-space sphere as shown in Fig. 2. The two strips are parallel circles on the wave normal sphere. The planes in which the strips lie are perpendicular to the grating vector $\mathbf{K}_{j l}$. If we select an inputoutput pair $(i, p)$, and if an additional input point $j$ is outside the bottom strip in Fig. 2, it will not produce cross talk to point $p$; if $j$ is within the bottom strip then cross talk will be eliminated if an output point is not placed at the same loca-

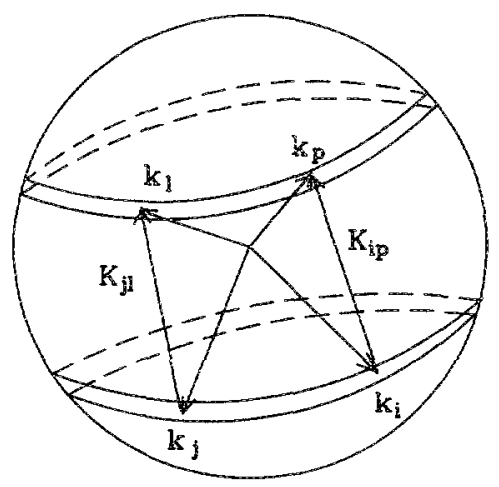

FIG. 2. k-space diagram illustrating the degeneracy of the gratings that connect points $(i, p)$ and $(j, l)$. 
tion as $l$ along the top strip. If these two criteria can be met for all input and output pixels, then first-order cross talk is completely eliminated. The required width of the strip in Fig. 2 is determined by several factors, including diffraction due to transverse aperture of the hologram, an effect we have not considered in this paper. The principal factor determining the width is the angular sensitivity of diffraction from a thick grating which is determined by the thickness of the crystal. The width of the strip that is required to satisfy Eq. (6) can be approximated for the purposes of this simplified exposition by $2 \pi / L \sin \theta$, where $\theta$ is the angle between $\mathrm{k}_{i}$ and $k_{p}$. This estimate is found by determining the angulat deviation of the incident and diffracted from the ideal Bragg condition, which will make $\eta_{i p l j}=0$ [see Eq. (3)].

In the above discussion we have specified the conditions that must be met so that each grating implements an independent interconnection in the crystal. The remaining task is to specify the arrangement of input and output pixels in the geometry of Fig. 1 so that the stated conditions are satisfied. We have developed an entire family of sampling patterns that accomplish this goal. ${ }^{3}$ Shown in Fig. 3 is one such sampling pattern for the input and output planes. To see why this is the case, consider first the gratings connecting two input points along the same row to two points at the same row at the output. These gratings can never be parallel to each other (i.e., fall within the same strip) because the horizontal ( $x$ direction in Fig. 1) difference in position between the input and the output locations is guaranteed to be different. If we consider two adjacent points in the same column at the input being connected to two adjacent points in the same column at the output, then we find that the two gratings connecting them are tilted with respect to each other in the $y-z$ plane (see the geometry of Fig. 1). In general, gratings conrecting points that are neither at the same row or column have gratings that are tilted with respect to each other in all three directions. The patterns in this example are drawn on a $9 \times 9=81$ rectangular grid and only $9^{3 / 2}=27$ points are utilized as input and output points in the input and output planes, resulting in a total number of connections $9^{3}=729$. In general, if the number of points available on a two-dimen-

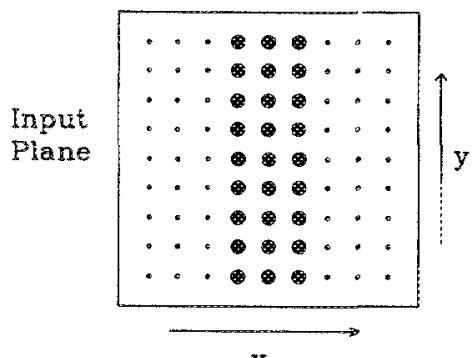

$x$

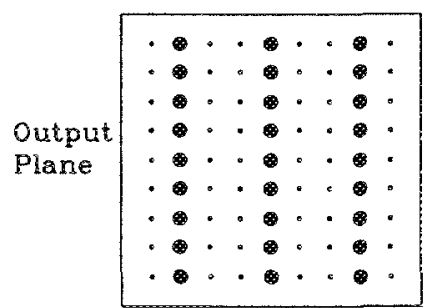

FIG. 3. Sampling patterns on $9 \times 9$ rectangular grids.

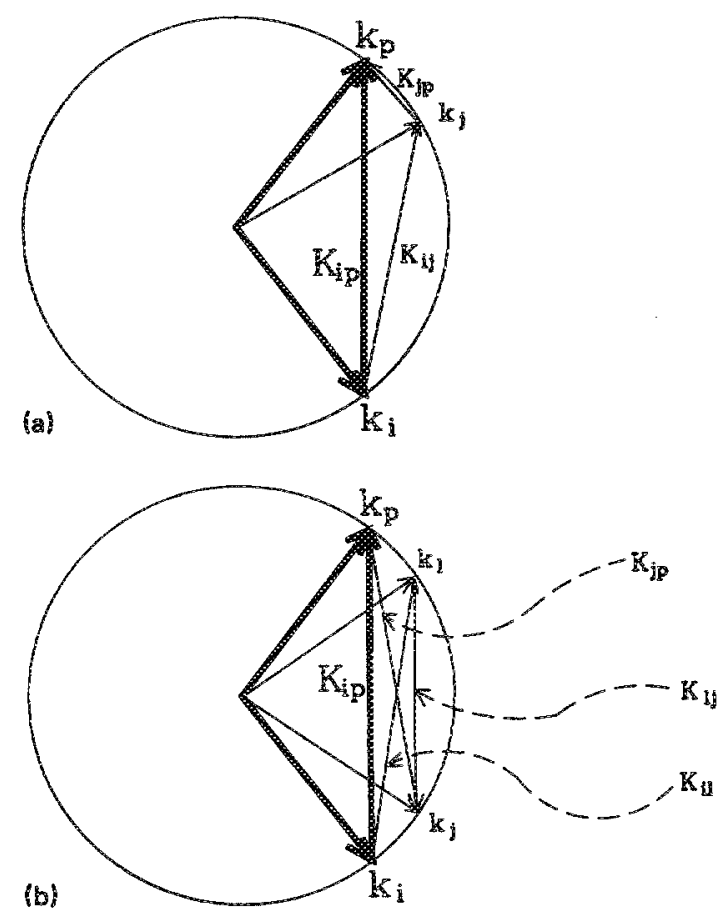

FIG. 4. Wave-vector matching diagram illustrating the mechanism through which (a) second-and (b) third-order cross talk is introduced at each output pixel $p$.

sional rectangular grid is $S^{2}$, then the number of pixels that are used for placement of neurons must be $N \leqslant S^{3 / 2}$ in order to ensure that the first-order cross talk can be eliminated. Equivalently, if we wish to have $N$ units in the input or output plane, then the number of resolvable points available must be $S^{2}=N^{4 / 3}$.

\section{HGHER ORDER CROSS TALK}

Second-order cross-talk effects result from light waves that are first diffracted by a grating from an input wave at pixel $i$, and then rediffracted by a second grating and are directed to the output pixel $p$. Therefore, two gratings are needed. All the second-order light waves resulting from diffraction by two intralayer gratings or two interlayer gratings are negligible because in the geometry of Fig. 1 they are phase mismatched and thus they do not contribute to second-order cross-talk effects. Therefore, the principal source of second-order cross talk is diffraction from the interlayer gratings followed by rediffraction from the intralayer gratings. Consider again an output pixel $p$ receiving light from an input pixel $i$ not directly through diffraction by the grating $\mathbf{K}_{i p}$ but through the intermediate step of diffraction first by an interlayer grating ${ }_{i j}$ followed by rediffraction by the grating $\mathbf{K}_{i p}$. This is depicted in a k-space diagram in Fig. 4 (a). Each input wave diffracts light to all $N^{4 / 3}$ output pixeis through interlayer gratings and at least $N$ pixeis are exactly Bragg matched to the $p$ th pixel through rediffraction of the intralayer gratings. Assuming that the overall diffraction efficiency is small, and therefore it neglects the depletion of the incident beam, we can easily calculate the second-order signal-to-noise ratio $\left(\mathrm{SNR}_{2}\right)$, defined as the ratio of the intensi-

(a)
Lee, Gu, and Psaltis

2193 
ty received at each output pixel due to the direct, first-order diffraction, divided by the total intensity received due to double diffraction:

$\mathrm{SNR}_{2}=\sum_{i}^{N} \eta_{p i} I_{p} \quad \frac{1}{4} \sum_{i}^{N} \sum_{j \neq p}^{N} \eta_{i j} \eta_{p j} I_{p} \approx 4 / N \eta_{i}$

In the above equation $\eta_{1}$ is the average diffraction efficiency for an intralayer grating. From Eq. (7) we see that it is desirable to minimize the strength of the intralayer gratings to eliminate the second-order cross talk. This can be accomplished by selecting a holographic recording medium in which low spatial frequencies are recorded weakly. This is, for instance, typical of gratings recorded in photorefractive crystals in the absence of an applied electric field, in which case the recording is done principally by diffusion of the carriers. In this case, gratings whose period is considerabiy longer than the diffusion length are not recorded effectively. As an example, if $\mathrm{KNbO}_{3}: \mathrm{Fe} 300 \mathrm{ppm}$ is utilized, ${ }^{6}$ the diffraction efficiency for $0.3-\mu \mathrm{m}$ fringe spacing is more than three orders of magnitude larger than the diffaction effciency for a fringe spacing of $2.6 \mu \mathrm{m}$. Hence, if the arrangements of input and output pixes are chosen such that the spatial frequency of the interlayer gratings is much higher than that of the intralayer gratings, then the effects of intralayer gratings can potentially be made negligible compared to third-order cross-talk effects, which we consider next.

Third-order cross talk arises when light originating from the $i$ th pixel is difracted by three separate interlayer gratings and is ulimately directed at the output pixel $p$. In order to calculate the total amount of third-order cross tak we need to determine the total number of three Braggmatched interlayer gratings whose vectorial sum is equal to $\mathrm{K}_{i p}$. An example of this condition is depicted in Fig. 4(b). This input beam in the direction of the ith pixel is Bragg matched to $N^{4 / 3}$ gratings ${ }^{7}$ and similarly, a beam diffracted towards the $l$ th output pixel is Bragg matched (and therefore rediffracted by) $N^{4 / 3}$ gratings. The ratio of the intensities due to first- and third-order diffaction is

$$
\mathrm{SNR}_{3}=\frac{\Sigma_{i}^{N} \eta_{p i} I_{i}}{\frac{1}{36} \sum_{i}^{N} \Sigma_{I}^{N^{4 / 3}} \sum_{j}^{N^{4 / 3}} \eta_{i i} \eta_{l j} \eta_{p j} \bar{I}_{i}} \approx \frac{36}{N^{8 / 3} \eta_{2}^{2}},
$$

where $\eta_{2}$ is the average diffraction efficiency of an interlayer grating. The conclusion that we might draw from Eq. (8) is that as the network become larger (i.e., $N$ increases) the signal-to-noise ratio deteriorates and therefore third-order cross talk imposes a limit on $N$. In fact, $\eta_{2}=\eta_{0} / N^{2}$ (Ref. 4 ) where $\eta_{0} \approx 1$ is the difraction efficiency obtained when only a single grating is recorded in the crystal. Substitution into
Eq. (8) reveals that $\mathrm{SNR}_{3}$ is proportional to $N^{4 / 3}$ which implies that for large networks third-order cross talk is not expected to be a serious concern.

\section{CONCLUSION}

We have used coupled mode analysis to derive a simple, approximate result for the conditions that must be met in order for each grating that is recorded in a volume hologram to implement an independent interconnection between two points in space. Since the number of gratings that can be stored in a volume medium is in the order of $V / \lambda^{3}$, , where $V$ is the volume of the crystal and $\lambda$ is the wavelength, the result reported here can make possible the design of optical networks with extremely high storage density. The effects of second-and third-order diffraction were calculated and it is shown that these effects can impose a limit on the number of units that can be interconnected with the same crystal, since the signal to noise ratio decreases monotonically as $N$ increases. There are of course several other factors, beyond the basic geometric constraints treated in this paper, which need to be taken into consideration in order to gain a complete understanding of the capabilities of volume holograms for implementing global interconnections. Most significantly, the effects of the recording mechanism and the limitations it imposes on the number of interconnections that a single hologram can implement ${ }^{4}$ must be addressed and combined with the results reported here. This will be the subject of a future pubiication.

\section{ACKMOMLEDGMENTS}

This work was supported at Caltech by Air Force Office of Scientific Research, the Army Research Office and DARPA, and at Polytechnic by the National Science Foundation EET-8810288 and the Center for Advanced Technology in Telecommunications.

'J. W. Goodman, F. J. Leonberger, S. Y. Kung, and R. A. A thale, Proc. IEFE 72, 850 ( 1984$)$.

${ }^{2} D$. Psaltis and P. Farhat, Opt. Lett. 10, 98 (1985).

'D. Psaltis, X. Gu, J. Yu, and H. Lee, Tech. Dig. Ser. 11, 129 (1987).

${ }^{4}$ D. Psaltis, D. Brady, and K. Wagner, Appl. Opt. 27, 1752 (1988).

${ }^{5}$ H. Kogelnik, Bell Syst. Tech. J. 48, 2909 (1969).

P. Gunter, Phys. Rep. 93, 199 (1982)

7D. Psaltis, X. Gu, H. Lee, and J. Yu (unpublished).

'P. Van Heerden, Appl. Opt. 2, 387 (1963). 